\title{
ON THE ALMOST CONVERGENCE OF DOUBLE SEQUENCES
}

\author{
DAVOR ButKović \\ University of Zagreb, Croatia
}

\begin{abstract}
We find necessary and sufficient conditions for transformations of double sequences almost convergent in the sense of G. H. Hardy to double sequences convergent in the sense of F. Pringsheim. The results extend the work of F. Móricz and B. E. Rhoades on transformations of sequences almost convergent in the Pringsheim's sense.
\end{abstract}

The first definitions and investigations of the convergence of double sequences are usually atributted to F. Pringsheim, who studied such sequences and series more than hundred years ago (see [1, p. 78]). Pringsheim defined what we call the $P$ limit and gave examples of convergence $(P$ convergence $)$ of double sequences with and without the usual convergence of rows and columns ([13, pp. 104-112]). G. H. Hardy ([6]) considered in more details the case of convergence of double sequences where, besides the existence of the $P$ limit, rows and columns converge. F. Móricz discovered an alternative approach to the Hardy convergence, which significantly influenced the whole theory ([9][11]; cf. [2]). Moreover, following G. G. Lorentz ([7]), F. Móricz and B. E. Rhoades found necessary and sufficient (N.S.) conditions under which $P$ almost convergent double sequences are transformed into $P$ convergent double sequences ([12, Theorem 1, p. 285]).

In a previous paper [3] the author of this article found conditions under which double sequences almost convergent in the Hardy $(H)$ sense are transformed into $P$ convergent double sequences. The results were not completely satisfactory because they were obtained under a uniformity condition inherited from the usual $H$ convergence (cf. [3, p. 252] and [14, p. 14]).

2010 Mathematics Subject Classification. 40A05, 40B05, $40 \mathrm{C} 05$.

Key words and phrases. Almost convergence, double sequences, Hardy convergence, transformation matrices. 
In this paper we have N.S. conditions for transformations of double sequences that $H$ almost converge without any further restriction. These conditions are the same as the Móricz-Rhoades conditions for transformations of $P$ almost convergent double sequences. Uniformities of row or column convergence now give various particular cases. Moreover, we have results on transformations of double sequences that $H$ almost converge only by columns or only by rows. For some results that can be reduced to results at [12] and [3] our approach here gives alternative proofs.

\section{The Hardy CONVERGEnCE}

Let us denote the set of all double sequences $x=\left(x_{i j}\right), i, j \in \mathbb{N}$, of complex (or real) numbers by $\mathbf{s}$. We consider $\left(x_{i j}\right)$ as the function on the $i j$-coordinate plane: $\left(x_{i j}\right)_{i \in \mathbb{N}}$ is the $j$-th row, and $\left(x_{i j}\right)_{j \in \mathbb{N}}$ is the $i$-th column of $x$. Let $\mathbf{b}$ be the set of all bounded double sequences from $\mathbf{s}$. A double sequence $x$ from $\mathbf{s}$ converges to $L$ if, for every $\varepsilon>0$ there exists $N_{\varepsilon} \in \mathbb{N}$ such that

$$
\left|x_{i j}-L\right|<\varepsilon \quad \text { if } i, j \geq N_{\varepsilon} .
$$

After $[1$, p. 78$]$ and $[6$, p. 88$]$ this kind of convergence we call the convergence in the sense of Pringsheim ( $P$ convergence). The limit $L$ is denoted by $\lim _{i j}$ and is called the $P$ limit (see [13, p. 103]). The set of all convergent double sequences from $\mathbf{s}$ is the class $\mathbf{c}$. Bounded and convergent double sequences form the class bc.

Starting from double series, both G. H. Hardy and later F. Móricz studied convergent double sequences with convergent rows and convergent columns. Such double sequences besides the $P$ limit $L=\lim _{i j} x_{i j}$ have row limits $L_{j}^{\prime}=\lim _{i} x_{i j}$ for every $j$ and column limits $L_{i}^{\prime \prime}=\lim _{j} x_{i j}$ for every $i$. If $\left(L_{j}^{\prime}\right)$ and $L$ exist we say that $\left(x_{i j}\right)$ converges in the Hardy $(H)$ sense by rows. If $\left(L_{i}^{\prime \prime}\right)$ and $L$ exist we have the $H$ convergence by columns. The class of double sequences that $H$ converges by rows and by columns form the class of $H$ convergent double sequences. It is denoted by rc (regularly convergent, after Hardy [6, p. 88] and Hamilton [4, p. 30]). The $P$ limit of double sequences from rc is also called the principal limit.

Double sequences from rc are bounded: $\mathbf{r c} \subseteq$ bc $([4$, p. 33]). Double sequences from rc with equal row and column limits we denote by rcr. For these double sequences $L_{j}^{\prime}=L_{i}^{\prime \prime}=L$. Subclasses of $\mathbf{c}$ of double sequences that converge to 0 are denoted by an $\mathbf{n}$ at the end: we have bcn, rcn and rcrn. The last class has all row limits and all column limits as well as the $P$ limit equal to 0 .

By the definition of rc,

$$
\lim _{i j} x_{i j}=\lim _{i} \lim _{j} x_{i j}=\lim _{j} \lim _{i} x_{i j} .
$$


The convergence of rows to $L_{j}^{\prime}$ and the convergence of columns to $L_{i}^{\prime \prime}$ is uniform with respect to $j$ 's, resp. to $i$ 's (see $[14$, Theorem 9, p. 14] and [4, Theorem 003, p. 34]). The convergence of rows and columns means that for every $\varepsilon>0$ there exist $N_{\varepsilon}^{\prime}, N_{\varepsilon}^{\prime \prime} \in \mathbb{N}$ such that for every $j$

$$
\left|x_{i j}-L_{j}^{\prime}\right|<\varepsilon \quad \text { if } \quad i \geq N_{\varepsilon}^{\prime},
$$

and for every $i$

$$
\left|x_{i j}-L_{i}^{\prime \prime}\right|<\varepsilon \quad \text { if } \quad j \geq N_{\varepsilon}^{\prime \prime} .
$$

A double sequence $x$ from $\mathbf{s}$ is almost convergent (a-convergent) to $L$ if

$$
\sigma_{p q}^{m n}=\frac{1}{p q} \sum_{i=m}^{m+p-1} \sum_{j=n}^{n+q-1} x_{i j},
$$

for $p, q \rightarrow \infty$ converges to $L$ uniformly with respect to $m$ and $n$. This means that for every $\varepsilon>0$ there exists $N_{\varepsilon} \in \mathbb{N}$ such that, for every $m, n \in \mathbb{N}$,

$$
\left|\sigma_{p q}^{m n}-L\right|<\varepsilon \quad \text { if } \quad p, q \geq N_{\varepsilon} .
$$

We denote $L$ by $\operatorname{Lim}_{i j} x_{i j}$ and consider it as the $P$ a-limit of $x$. The set of all $a$-convergent double sequences we denote by ac.

A single sequence $\left(x_{i}\right) a$-converges if $\frac{1}{p} \sum_{i=m}^{p+m-1} x_{i}$ converges when $p \rightarrow \infty$ uniformly with respect to $m \in \mathbb{N}$. This notion was introduced by G. G. Lorentz ([7]). It was extended to double sequences i. e. to the class ac by F. Móricz and B. E. Rhoades ([12]). Among others, they proved bc $\subset \mathbf{a c} \subset \mathbf{b}$ ([12, pp. 283-4]).

The double sequence $x H$ a-converges by rows if every row $a$-converges to $L_{j}^{\prime}=\operatorname{Lim}_{i} x_{i j}$ and the $P a$-limit $L$ of $x$ exists (cf. Lemma 1.1). In such a case $L$ is the principal $a$-limit, and $L_{j}^{\prime}$ are row $a$-limits. Column $a$-limits and the $H$ a-convergence by columns are similarly defined. The double sequence $x H a$-converges if it is $H a$-convergent by rows and columns to the $P a$-limit. The class of $H a$-convergent sequences are denoted by rac. The racn is the subclass of rac with the $P a$-limit 0 . The racr is a subclass of rac with equal $L, L_{j}^{\prime}$ and $L_{i}^{\prime \prime}$ for every $i, j$. If $L=L_{j}^{\prime}=L_{i}^{\prime \prime}=0$ we have the class racrn.

Lemma 1.1 ([2, Theorem 1, p. 132]).Let $\left(x_{i j}\right)$ P a-converge to $\operatorname{Lim}_{i j} x_{i j}=$ $L$, and, moreover, let every row $\left(x_{i j}\right)_{i \in \mathbb{N}}$ a-converge to $\operatorname{Lim}_{i} x_{i j}=L_{j}^{\prime}$. Then $\left(L_{j}^{\prime}\right)$ a-converges and

$$
\operatorname{Lim}_{j} \operatorname{Lim}_{i} x_{i j}=\operatorname{Lim}_{i j} x_{i j} .
$$

Similarly, if the $P$ a-limit and all column a-limits exist,

$$
\operatorname{Lim}_{i} \operatorname{Lim}_{j} x_{i j}=\operatorname{Lim}_{i j} x_{i j} .
$$

COROllary 1.2 ([2, p. 132]). Let a-limits of rows, a-limits of columns and the principal a-limit of the double sequence $x$ exist. Then

$$
\operatorname{Lim}_{i j} x_{i j}=\operatorname{Lim}_{j} \operatorname{Lim}_{i} x_{i j}=\operatorname{Lim}_{i} \operatorname{Lim}_{j} x_{i j} .
$$


This corollary excludes the existence of the $P a$-limit if $a$-limits of $L_{j}^{\prime}$ and of $L_{i}^{\prime \prime}$ exist and are unequal (for $P$ limits cf. [13, p. 107]).

By row-column uniformities we have a partial inverse of Lemma 1.1.

LemMA 1.3. Let rows of $x=\left(x_{i j}\right)$ a-converge to $L_{j}^{\prime}$ uniformly, and let the sequence $\left(L_{j}^{\prime}\right)$ a-converge. Then the $P$ a-limit of $x$ exists and $x H$ a-converges by rows. Similarly for uniform a-convergence of columns.

ProOF. By the uniform $a$-convergence of rows

$$
\left|\frac{1}{p} \sum_{i=m}^{m+p-1} x_{i j}-L_{j}^{\prime}\right| \leq \frac{\varepsilon}{2} \quad \text { if } \quad p \geq N_{\frac{\varepsilon}{2}}^{\prime}
$$

for every $j \in \mathbb{N}$. By the $a$-convergence of row $a$-limits,

$$
\left|\frac{1}{q} \sum_{j=n}^{n+q-1} L_{j}^{\prime}-\operatorname{Lim}_{j} L_{j}^{\prime}\right| \leq \frac{\varepsilon}{2} \quad \text { if } \quad q \geq N_{\frac{\varepsilon}{2}}^{\prime} .
$$

Therefore,

$$
\begin{aligned}
& \left|\frac{1}{q} \sum_{j=n}^{n+q-1} \frac{1}{p} \sum_{i=m}^{m+p-1} x_{i j}-\operatorname{Lim}_{j} L_{j}^{\prime}\right| \\
& \quad \leq \frac{1}{q} \sum_{j=n}^{n+q-1}\left|\frac{1}{p} \sum_{i=m}^{m+p-1} x_{i j}-L_{j}^{\prime}\right|+\left|\frac{1}{q} \sum_{j=n}^{n+q-1} L_{j}^{\prime}-\operatorname{Lim}_{j} L_{j}^{\prime}\right| \\
& \quad \leq \frac{\varepsilon}{2}+\frac{\varepsilon}{2}=\varepsilon .
\end{aligned}
$$

This means that

$$
\operatorname{Lim}_{j} \operatorname{Lim}_{i} x_{i j}=\operatorname{Lim}_{i j} x_{i j}
$$

\section{TRANSFORMATION CONDITIONS}

Let $A=\left[a_{i j}^{k l}\right], i, j, k, l \in \mathbb{N}$, be a doubly infinite matrix of complex (or real) numbers. A double sequence $x=\left(x_{i j}\right) \in \mathbf{s}$ is transformed into a double sequence $A x=y=\left(y^{k l}\right) \in \mathbf{s}$ by

$$
y^{k l}=\sum_{i=1}^{\infty} \sum_{j=1}^{\infty} a_{i j}^{k l} x_{i j}
$$

if the double series (2.1) $P$ converges for every $k, l \in \mathbb{N}$. It means that the partial sums $\sum_{i=1}^{r} \sum_{j=1}^{s}$ have the $P$ limit if $r, s \rightarrow \infty([13,(3)$ p. 113]). The partial sums for $A$ are called $A$-means ([12, p. 284]). The matrix $A$ is bounded-regular if every bounded and $P$ convergent double sequence $\left(x_{i j}\right)$ is transformed by the bounded set of $A$-means to the $P$ convergent double 
sequence $\left(y^{k l}\right)$ with the limit equal to the limit of $\left(x_{i j}\right)$. The matrix $A$ is strongly regular if every $P a$-convergent double sequence $\left(x_{i j}\right)$ is transformed to the $P$ convergent $\left(y^{k l}\right)$ with the limit equal to the $a$-limit of $\left(x_{i j}\right)$, and the $A$-means are also bounded ( $y$ P converges boundedly). The above definitions extends to double sequences the notion of regularity and of strong regularity of single sequences (see [7, pp. 171, 176]).

We consider bounded transformations of classes which are between rcrn and $\mathbf{b}$. Necessary conditions that $\mathbf{r c r n}$ transforms to $\mathbf{b}$ include the existence of $C$ such that

$$
\sum_{i=1}^{\infty} \sum_{j=1}^{\infty}\left|a_{i j}^{k l}\right| \leq C<+\infty
$$

for every $k, l \in \mathbb{N}([4,5$., p. 42]). This implies that for every bounded $y$ the series (2.1) which defines $\left(y^{k l}\right)$ converges absolutely. Because our $y$ are at least bounded, conditions on $A$ always include (with the notation (2.4) below), $(v) \in \mathbf{b}$.

We use the notation

$$
\begin{aligned}
& \triangle_{10} a_{i j}^{k l}=a_{i j}^{k l}-a_{i+1, j}^{k l}, \quad \triangle_{01} a_{i j}^{k l}=a_{i j}^{k l}-a_{i, j+1}^{k l}, \\
& \square a_{i j}^{k l}=a_{i j}^{k l}-a_{i+1, j}^{k l}-a_{i, j+1}^{k l}+a_{i+1, j+1}^{k l} .
\end{aligned}
$$

Series are denoted by $\sum_{i}=\sum_{i=1}^{\infty}, \sum_{j}=\sum_{j=1}^{\infty}$. Also, we give notations for sequences derived from $\left[a_{i j}^{k l}\right]$. The list is a slightly enlarged list ([3, p. 254]). The only change is that instead of $\left(i i_{\triangle}^{\prime}\right)$ we write $\left(i i_{\triangle}\right)$. Also, we have $a_{i j}$ for $a_{i j}^{k l}$.

(i) $(\forall i, j) a_{i j}$

$$
\text { (ii) } \begin{gathered}
\left\{\begin{array}{lll}
(\forall j) & \sum_{i} a_{i j} \\
(\forall i) & \sum_{j} a_{i j} & \left(i i_{\triangle}\right)
\end{array}\right. \\
(\text { iii })\left\{\begin{array}{l}
(\forall j) \\
(\forall i) \sum_{i} \triangle_{j} \triangle_{01} a_{i j} a_{i j}
\end{array}\right. \\
\begin{array}{l}
\sum_{j} \sum_{i} a_{i j} \\
\sum_{i} \sum_{j} a_{i j}
\end{array}
\end{gathered}
$$

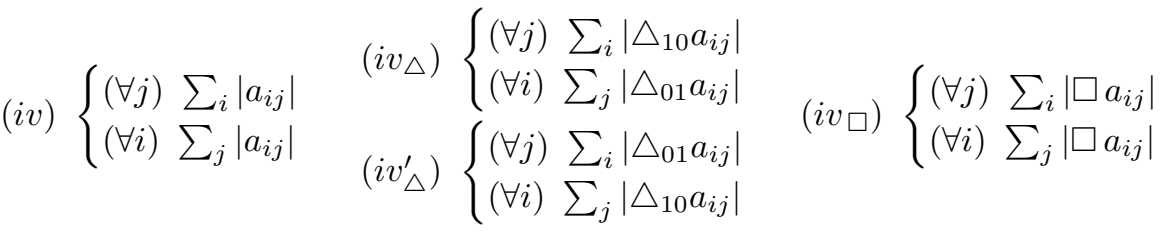
(v) $\sum_{i} \sum_{j}\left|a_{i j}\right| \quad\left(v_{\triangle}\right)\left\{\begin{array}{l}\sum_{i} \sum_{j}\left|\triangle_{01} a_{i j}\right| \\ \sum_{i} \sum_{j}\left|\triangle_{10} a_{i j}\right|\end{array} \quad\left(v_{\square}\right) \sum_{i} \sum_{j}\left|\square a_{i j}\right|\right.$ 
(vi) $\left\{\begin{array}{l}\sum_{j}\left|\sum_{i} a_{i j}\right| \\ \sum_{i}\left|\sum_{j} a_{i j}\right|\end{array} \quad\left(v i_{\triangle}\right)\left\{\begin{array}{l}\sum_{j}\left|\sum_{i} \triangle_{01} a_{i j}\right| \\ \sum_{i}\left|\sum_{j} \triangle_{10} a_{i j}\right|\end{array} \quad\left(v i_{\square}\right)\left\{\begin{array}{l}\sum_{j}\left|\sum_{i} \square a_{i j}\right| \\ \sum_{i}\left|\sum_{j} \square a_{i j}\right|\end{array}\right.\right.\right.$

For members of (2.4) which have $\{$ and two lines, the first and the second line are denoted by ()$_{1}$ and ()$_{2}$. E. g., $\left(v i_{\triangle}\right)_{2} \in$ bcn means $\sum_{i}\left|\sum_{j} \triangle_{10} a_{i j}^{k l}\right| \in$ bcn; $\left(v i_{\triangle}\right) \in$ ben means that $\left(v i_{\triangle}\right)_{1}$ and $\left(v i_{\triangle}\right)_{2}$ belong to bcn.

Lemma 2.1. (cf. $[5,(17)$ p. 279]). Let $(v) \in$ b. Then

$$
\sum_{i=1}^{\infty}\left|\triangle_{10} a_{i t}^{k l}\right| \leq \sum_{i=1}^{\infty} \sum_{j=t}^{\infty}\left|\square a_{i j}^{k l}\right|, \quad \sum_{j=1}^{\infty}\left|\triangle_{01} a_{t j}^{k l}\right| \leq \sum_{i=t}^{\infty} \sum_{j=1}^{\infty}\left|\square a_{i j}^{k l}\right| .
$$

Proof. The $(v) \in \mathbf{b}$ implies $\left(v_{\square}\right) \in \mathbf{b}$ and

$$
\sum_{i=1}^{\infty}\left|\sum_{j=t}^{\infty} \square a_{i j}^{k l}\right|=\sum_{i=1}^{\infty}\left|\sum_{j=t}^{\infty}\left(\triangle_{10} a_{i j}^{k l}-\triangle_{10} a_{i, j+1}^{k l}\right)\right|=\sum_{i=1}^{\infty}\left|\triangle_{10} a_{i t}^{k l}\right| .
$$

Therefore,

$$
(\forall t) \sum_{i=1}^{\infty}\left|\triangle_{10} a_{i t}^{k l}\right| \leq \sum_{i=1}^{\infty} \sum_{j=t}^{\infty}\left|\square a_{i j}^{k l}\right|
$$

Similarly for $\triangle_{01}$.

By this Lemma, if $(v) \in \mathbf{b}$,

$$
\left(v_{\square}\right) \in \mathbf{b c n} \Rightarrow\left\{\begin{array}{l}
\left(i v_{\triangle}\right)_{1} \in \mathbf{b c n} \\
\left(i v_{\triangle}\right)_{2} \in \mathbf{b c n}
\end{array}\right.
$$

Lemma 2.2 (cf. [8, p. 806, (8) and (3)]). Let $(v) \in$ b. Then

$$
\left|\sum_{i=1}^{\infty} a_{i j}^{k l}\right| \leq \sum_{j=1}^{\infty}\left|\sum_{i=1}^{\infty} \triangle_{01} a_{i j}^{k l}\right|, \quad\left|\sum_{j=1}^{\infty} a_{i j}^{k l}\right| \leq \sum_{i=1}^{\infty}\left|\sum_{j=1}^{\infty} \triangle_{10} a_{i j}^{k l}\right| .
$$

Proof. With $a_{i j}=a_{i j}^{k l}$ and $C$ from (2.2),

$$
\sum_{j=1}^{\infty}\left|\sum_{i=1}^{\infty}\left(a_{i j}-a_{i, j+1}\right)\right| \leq 2 \sum_{i=1}^{\infty} \sum_{j=1}^{\infty}\left|a_{i j}\right| \leq 2 C .
$$

Let

$$
\sum_{j=1}^{\infty}\left|\sum_{i=1}^{\infty}\left(a_{i j}-a_{i, j+1}\right)\right|=D
$$

By

$$
\left|\sum_{i=1}^{\infty} a_{i 1}-\sum_{i=1}^{\infty} a_{i 2}\right|+\cdots+\left|\sum_{i=1}^{\infty} a_{i t}-\sum_{i=1}^{\infty} a_{i, t+1}\right| \leq D
$$


we have

$$
\begin{gathered}
\left|\left(\sum_{i=1}^{\infty} a_{i 1}-\sum_{i=1}^{\infty} a_{i 2}\right)+\cdots+\left(\sum_{i=1}^{\infty} a_{i t}-\sum_{i=1}^{\infty} a_{i, t+1}\right)\right| \\
=\left|\sum_{i=1}^{\infty} a_{i 1}-\sum_{i=1}^{\infty} a_{i, t+1}\right| \leq D .
\end{gathered}
$$

If $t \rightarrow \infty,\left|\sum_{i} a_{i 1}\right| \leq D$. Starting with $\sum_{j=s}^{\infty}$ we have $\left|\sum_{i} a_{i s}\right| \leq D$ for $s=2,3, \ldots$ Therefore

$$
(\forall j)\left|\sum_{i=1}^{\infty} a_{i j}^{k l}\right| \leq \sum_{j=1}^{\infty}\left|\sum_{i=1}^{\infty} \triangle_{01} a_{i j}^{k l}\right|
$$

and similarly for $\triangle_{10}$. By the notation $(2.4)$, if $(v) \in \mathbf{b}$,

$$
\left(v i_{\triangle}\right)_{1} \in \mathbf{b c n} \Rightarrow(i i)_{1} \in \mathbf{b c n}, \quad\left(v i_{\triangle}\right)_{2} \in \mathbf{b c n} \Rightarrow(i i)_{2} \in \mathbf{b c n}
$$

(cf. also Remark 2.8).

Lemma 2.3. Let $(v) \in \mathbf{b}$. Then

$$
\begin{aligned}
& (\forall i, j) \quad\left|a_{i j}^{k l}\right| \leq \sum_{j=1}^{\infty}\left|\triangle_{01} a_{i j}^{k l}\right|, \\
& (\forall i, j) \quad\left|a_{i j}^{k l}\right| \leq \sum_{i=1}^{\infty}\left|\triangle_{10} a_{i j}^{k l}\right|
\end{aligned}
$$

and therefore

$$
\begin{aligned}
& \left(i v_{\triangle}\right)_{2} \in \mathbf{b c n} \Rightarrow(i) \in \mathbf{b c n}, \\
& \left(i v_{\triangle}\right)_{1} \in \mathbf{b c n} \Rightarrow(i) \in \mathbf{b c n} .
\end{aligned}
$$

Proof. By the proof of Lemma 2.2 with $a_{i j}^{k l}$ instead of $\sum_{i} a_{i j}^{k l}$ for every $i$ we have the first inequality. The second inequality is obtained in the same way.

The next corollary follows from Lemma 2.1 and Lemma 2.3.

Corollary 2.4. Let $(v) \in \mathbf{b}$. Then

$$
\left(v_{\square}\right) \in \mathbf{b c n} \Rightarrow(i) \in \mathbf{b c n} .
$$

By Lemma 2.3

$$
(\forall j) \quad \sum_{i=1}^{\infty}\left|a_{i j}^{k l}\right| \leq \sum_{i=1}^{\infty} \sum_{j=1}^{\infty}\left|\triangle_{01} a_{i j}^{k l}\right|, \quad(\forall i) \quad \sum_{j=1}^{\infty}\left|a_{i j}^{k l}\right| \leq \sum_{i=1}^{\infty} \sum_{j=1}^{\infty}\left|\triangle_{10} a_{i j}^{k l}\right|,
$$

which gives 
Corollary 2.5. Let $(v) \in \mathbf{b}$. Then

$$
\left(v_{\triangle}\right)_{1} \in \mathbf{b c n} \Rightarrow(i v)_{1} \in \mathbf{b c n}, \quad\left(v_{\triangle}\right)_{2} \in \mathbf{b c n} \Rightarrow(i v)_{2} \in \mathbf{b c n} .
$$

Remark 2.6. By Corollary 2.5, $(v) \in \mathbf{b}$ and the Móricz-Rhoades $\left(v_{\triangle}\right) \in$ bcn are N.S. for transformations of double sequences from racrn to double $P$ convergent null-sequences. These conditions include $(i),(i v) \in \mathbf{b c n}$ (cf. [12, pp. 286-287]).

Lemma 2.7. Let $(v) \in \mathbf{b}$. Then

$$
\begin{aligned}
& \sum_{i=1}^{\infty} \sum_{j=1}^{\infty}\left|\square a_{i j}^{k l}\right| \leq 2 \sum_{i=1}^{\infty} \sum_{j=1}^{\infty}\left|\triangle_{01} a_{i j}^{k l}\right|, \\
& \sum_{i=1}^{\infty} \sum_{j=1}^{\infty}\left|\square a_{i j}^{k l}\right| \leq 2 \sum_{i=1}^{\infty} \sum_{j=1}^{\infty}\left|\triangle_{10} a_{i j}^{k l}\right| .
\end{aligned}
$$

Therefore

$$
\begin{aligned}
& \left(v_{\triangle}\right)_{1} \in \mathbf{b c n} \Rightarrow \quad\left(v_{\square}\right) \in \mathbf{b c n}, \\
& \left(v_{\triangle}\right)_{2} \in \mathbf{b c n} \Rightarrow \quad\left(v_{\square}\right) \in \mathbf{b c n} .
\end{aligned}
$$

The results of lemmas are summarized in (2.23). Arrows give implications if members of (2.23) belong to bcn and if to every initial condition the $(v) \in \mathbf{b}$ is added. E. g. $\left(v_{\triangle}\right)_{2} \rightarrow\left(v i_{\triangle}\right)_{2}$ means that $\sum_{i} \sum_{j}\left|\triangle_{10} a_{i j}^{k l}\right| \in$ bcn and $\sum_{i} \sum_{j}\left|a_{i j}^{k l}\right| \in \mathbf{b}$ imply $\sum_{i}\left|\sum_{j} \triangle_{10} a_{i j}^{k l}\right| \in \mathbf{b c n}$.

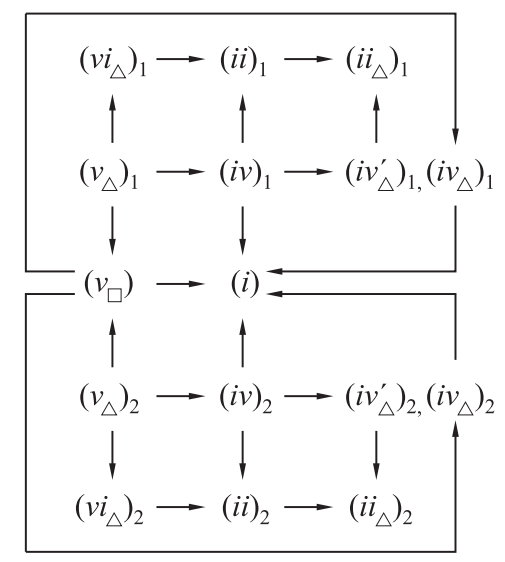

REMARK 2.8. The schema (2.23) neglects uniformities that in some cases follow from lemmas above. For example, by $(2.20)$ we have that $\left(v_{\triangle}\right)_{1} \in \mathbf{b c n}$ implies $(i v)_{1} \in$ bcn for every $j$, but also that the convergence by $(k, l)$ is uniform with respect to $j$. Similarly for estimates by $(v),\left(v i_{\triangle}\right),\left(v_{\square}\right)$, etc. 
Remark 2.9. Instead of $(v) \in \mathbf{b}$, many papers start with $\left(v_{\square}\right) \in \mathbf{b}$ (and the notation $\triangle_{11}$ for our $\square$ ). Hardy ([6, 4.(2), p. 89-90]) has a bounded variation for bounded $\left(v_{\square}\right)$ and $\left(i v_{\triangle}\right)$. Mears $([8$, p. 805]) defines absolutely convergent sequences by $\left(v_{\square}\right) \in \mathbf{b}$, with classes $a \mathbf{c}$, arc, etc. Hamilton ([5, p. 276]) has bounded $\sum_{i, j=1}^{\infty}\left|\square a_{i j}\right|$ jointly with other conditions.

\section{Almost COnvergence By Rows And COLUmns} of

The $a$-convergence by rows and columns can be described by $\sigma_{p q}^{m n}$ because

$$
\frac{1}{p} \sum_{i=m}^{m+p-1} x_{i j}=\sigma_{p 1}^{m j}, \quad \frac{1}{q} \sum_{j=n}^{n+q-1} x_{i j}=\sigma_{1 q}^{i n} .
$$

The $j$-th row $a$-converges to $L_{j}^{\prime}$ if, for every $\varepsilon>0$ there exists $N_{\varepsilon}^{\prime}(j)$ such that, for $p \geq N_{\varepsilon}^{\prime}(j)$ and every $m$,

$$
\left|\sigma_{p 1}^{m j}-L_{j}^{\prime}\right| \leq \varepsilon
$$

The $i$-th column $a$-converges to $L_{i}^{\prime \prime}$ if, for every $\varepsilon>0$ there exists $N_{\varepsilon}^{\prime \prime}(i)$ such that, for $q \geq N_{\varepsilon}^{\prime \prime}(i)$ and every $n$,

$$
\left|\sigma_{1 q}^{i n}-L_{i}^{\prime \prime}\right| \leq \varepsilon .
$$

If $x \mathrm{Ha}$-converges by rows, $L_{j}^{\prime} a$-converges to the $P a$-limit $L$, and for every $\varepsilon>0$, there exists $N_{\varepsilon}^{\prime}$ such that, for $q \geq N_{\varepsilon}^{\prime}$,

$$
\left|\frac{1}{q} \sum_{j=n}^{n+q-1} L_{j}^{\prime}-L\right| \leq \varepsilon
$$

If $x H a$-converges by columns there exists $N_{\varepsilon}^{\prime \prime}$ such that, for $p \geq N_{\varepsilon}^{\prime \prime}$,

$$
\left|\frac{1}{p} \sum_{i=m}^{m+p-1} L_{i}^{\prime \prime}-L\right| \leq \varepsilon
$$

Lemma 3.1. Let $x H$ a-converges to $L$. Then, with $N_{\varepsilon}$ from (1.6),

$$
N_{\varepsilon}^{\prime}, N_{\varepsilon}^{\prime \prime} \leq N_{\varepsilon} .
$$

Proof. Because of (1.6),

$$
p, q \geq N_{\varepsilon} \Rightarrow\left|\frac{1}{p q} \sum_{i=m}^{m+p-1} \sum_{j=n}^{n+q-1} x_{i j}-L\right| \leq \varepsilon .
$$

Increasing $p$ we obtain (3.4), and increasing $q$ we obtain (3.5). As a result, we have (3.6). 
For $x \in$ racrn, which has at least one $x_{i j} \neq 0$, it follows that $N_{\varepsilon}^{\prime}=N_{\varepsilon}^{\prime \prime}=0$ and $N_{\varepsilon}>0$. If $x$ has equal rows convergent to 0 and at least one $x_{i j} \neq 0$, we have $N_{\varepsilon}^{\prime}=N_{\varepsilon}>0, N_{\varepsilon}^{\prime \prime}=0$.

In what follows we use abbreviations

$$
\begin{aligned}
\Sigma_{R C}^{k l} & =\sum_{m=1}^{\infty} \sum_{n=1}^{\infty} a_{m n}^{k l} \frac{1}{p q} \sum_{i=m}^{m+p-1} \sum_{j=n}^{n+q-1} x_{i j}=\sum_{m=1}^{\infty} \sum_{n=1}^{\infty} a_{m n}^{k l} \sigma_{p q}^{m n}, \\
\Sigma_{R}^{k l} & =\sum_{m=1}^{\infty} \sum_{j=1}^{\infty} a_{m j}^{k l} \frac{1}{p} \sum_{i=m}^{m+p-1} x_{i j}=\sum_{m=1}^{\infty} \sum_{j=1}^{\infty} a_{m j}^{k l} \sigma_{p 1}^{m j}, \\
\sum_{C}^{k l} & =\sum_{i=1}^{\infty} \sum_{n=1}^{\infty} a_{i n}^{k l} \frac{1}{q} \sum_{j=n}^{n+q-1} x_{i j}=\sum_{i=1}^{\infty} \sum_{n=1}^{\infty} a_{i n}^{k l} \sigma_{1 q}^{i n} .
\end{aligned}
$$

With $L$ as a $P$ a-limit of $x$,

$$
\begin{aligned}
y^{k l}-L= & \left(\Sigma_{R C}^{k l}-y^{k l}\right)-\left(\Sigma_{R}^{k l}-y^{k l}\right)-\left(\sum_{C}^{k l}-y^{k l}\right) \\
& -\left(\sum_{R C}^{k l}-L\right)+\left(\Sigma_{R}^{k l}-L\right)+\left(\Sigma_{C}^{k l}-L\right) .
\end{aligned}
$$

For $L=0$ as well as for $L \neq 0$,

(3.10) $y^{k l}+\Sigma_{R C}^{k l}-\sum_{R}^{k l}-\sum_{C}^{k l}=\left(\sum_{R C}^{k l}-y^{k l}\right)-\left(\sum_{R}^{k l}-y^{k l}\right)-\left(\sum_{C}^{k l}-y^{k l}\right)$.

The subclass of rac with uniformly $a$-convergent rows and columns is denoted by rac un. This means that (3.2) holds uniformly with respect of $j$, as well as (3.3) with respect of $i$. The class racrn un has uniform $a$ convergent rows and columns to 0 . These classes are in [3] denoted by arc, resp. by arcrn.

Lemma 3.2. Let $x$ be bounded. For every $p, q \in \mathbb{N}, \varepsilon>0$ and $k, l \rightarrow \infty$ sufficient conditions on $A=\left[a_{i j}^{k l}\right]$ such that

$$
Y^{k l}=\left(\Sigma_{R C}^{k l}-y^{k l}\right)-\left(\Sigma_{R}^{k l}-y^{k l}\right)-\left(\Sigma_{C}^{k l}-y^{k l}\right)
$$

is by absolute value less of $\varepsilon$ are

$$
(v) \in \mathbf{b} ; \quad\left(v_{\square}\right) \in \mathbf{b c n} .
$$

Proof. We change the order of summation of the left-hand-side of (3.10). Instead of $a_{i j}^{k l}$ we write $a_{i j}$. If the sum runs over an index that does not appear among indexes, the argument of the sum is constant. For example, $\sum_{m=i-p+1}^{i} a_{i j}=p a_{i j}$.

$$
y^{k l}=\sum_{i=1}^{p-1} \sum_{j=1}^{q-1} x_{i j} a_{i j}+\frac{1}{p} \sum_{i=p}^{\infty} \sum_{j=1}^{q-1} x_{i j} \sum_{m=i-p+1}^{i} a_{i j}
$$




$$
\begin{aligned}
& +\frac{1}{q} \sum_{i=1}^{p-1} \sum_{j=q}^{\infty} x_{i j} \sum_{n=j-q+1}^{j} a_{i j} \\
& +\frac{1}{p q} \sum_{i=p}^{\infty} \sum_{j=q}^{\infty} x_{i j} \sum_{m=i-p+1}^{i} \sum_{n=j-q+1}^{j} a_{i j},
\end{aligned}
$$

(3.14) $\Sigma_{R}^{k l}=\frac{1}{p} \sum_{i=1}^{p-1} \sum_{j=1}^{q-1} x_{i j} \sum_{m=1}^{i} a_{m j}+\frac{1}{p} \sum_{i=p}^{\infty} \sum_{j=1}^{q-1} x_{i j} \sum_{m=i-p+1}^{i} a_{m j}$

$$
\begin{aligned}
& +\frac{1}{p q} \sum_{i=1}^{p-1} \sum_{j=q}^{\infty} x_{i j} \sum_{m=1}^{i} \sum_{n=j-q+1}^{j} a_{m j} \\
& +\frac{1}{p q} \sum_{i=p}^{\infty} \sum_{j=q}^{\infty} x_{i j} \sum_{m=i-p+1}^{i} \sum_{n=j-q+1}^{j} a_{m j},
\end{aligned}
$$

(3.15) $\Sigma_{C}^{k l}=\frac{1}{q} \sum_{i=1}^{p-1} \sum_{j=1}^{q-1} x_{i j} \sum_{n=1}^{j} a_{i n}+\frac{1}{p q} \sum_{i=p}^{\infty} \sum_{j=1}^{q-1} x_{i j} \sum_{m=i-p+1}^{i} \sum_{n=1}^{j} a_{i n}$

$$
\begin{aligned}
& +\frac{1}{q} \sum_{i=1}^{p-1} \sum_{j=q}^{\infty} x_{i j} \sum_{n=j-q+1}^{j} a_{i n} \\
& +\frac{1}{p q} \sum_{i=p}^{\infty} \sum_{j=q}^{\infty} x_{i j} \sum_{m=i-p+1}^{i} \sum_{n=j-q+1}^{j} a_{i n},
\end{aligned}
$$

(3.16) $\Sigma_{R C}^{k l}=\frac{1}{p q} \sum_{i=1}^{p-1} \sum_{j=1}^{q-1} x_{i j} \sum_{m=1}^{i} \sum_{n=1}^{j} a_{m n}+\frac{1}{p q} \sum_{i=p}^{\infty} \sum_{j=1}^{q-1} x_{i j} \sum_{m=i-p+1}^{i} \sum_{n=1}^{j} a_{m n}$

$$
\begin{aligned}
& +\frac{1}{p q} \sum_{i=1}^{p-1} \sum_{j=q}^{\infty} x_{i j} \sum_{m=1}^{i} \sum_{n=j-q+1}^{j} a_{m n} \\
& +\frac{1}{p q} \sum_{i=p}^{\infty} \sum_{j=q}^{\infty} x_{i j} \sum_{m=i-p+1}^{i} \sum_{n=j-q+1}^{j} a_{m n} .
\end{aligned}
$$


Grouping the corresponding terms of (3.13)-(3.16) we get

$$
\begin{aligned}
y^{k l}- & \sum_{R}^{k l}-\Sigma_{C}^{k l}+\sum_{R C}^{k l} \\
= & \sum_{i=1}^{p-1} \sum_{j=1}^{q-1} x_{i j} a_{i j}-\frac{1}{p} \sum_{i=1}^{p-1} \sum_{j=1}^{q-1} x_{i j} \sum_{m=1}^{i} a_{m j} \\
& -\frac{1}{q} \sum_{i=1}^{p-1} \sum_{j=1}^{q-1} x_{i j} \sum_{n=1}^{j} a_{i n}+\frac{1}{p q} \sum_{i=1}^{p-1} \sum_{j=1}^{q-1} x_{i j} \sum_{m=1}^{i} \sum_{n=1}^{j} a_{m n} \\
& +\sum_{i=p}^{\infty} \sum_{j=1}^{q-1} x_{i j}\left[\frac{1}{p} \sum_{m=i-p+1}^{i}\left(a_{i j}-a_{m j}\right)-\frac{1}{p q} \sum_{m=i-p+1}^{i} \sum_{n=1}^{j}\left(a_{i n}-a_{m n}\right)\right] \\
& +\sum_{i=1}^{p-1} \sum_{j=q}^{\infty} x_{i j}\left[\frac{1}{q} \sum_{n=j-q+1}^{j}\left(a_{i j}-a_{i n}\right)-\frac{1}{p q} \sum_{m=1}^{i} \sum_{n=j-q+1}^{j}\left(a_{m j}-a_{m n}\right)\right] \\
& +\sum_{i=p}^{\infty} \sum_{j=q}^{\infty} x_{i j}\left[\frac{1}{p q} \sum_{m=i-p+1}^{i} \sum_{n=j-q+1}^{j}\left(a_{i j}-a_{m j}-a_{i n}+a_{m n}\right)\right] .
\end{aligned}
$$

Differences of terms of $A$ are finite sums of $a_{\mu \nu}, \triangle_{10} a_{\mu \nu}, \triangle_{01} a_{\mu \nu}$ and $\square a_{\mu \nu}$ by

$$
\begin{aligned}
& a_{m n}-a_{i n}=\sum_{\mu=m}^{i-1} \triangle_{10} a_{\mu n}, \quad a_{m n}-a_{m j}=\sum_{\nu=n}^{j-1} \triangle_{01} a_{m \nu}, \\
& a_{m j}-a_{i j}=\sum_{\mu=m}^{i-1} \triangle_{10} a_{\mu j}, \quad a_{i n}-a_{i j}=\sum_{\nu=n}^{j-1} \triangle_{01} a_{i \nu}, \\
& a_{m n}-a_{i n}-a_{m j}+a_{i j}=\sum_{\mu=m}^{i-1} \sum_{\nu=n}^{j-1} \square a_{\mu \nu} .
\end{aligned}
$$

Therefore, increasing $k$ and $l$ for every $\varepsilon>0$ the estimate

$$
\left|y^{k l}-\Sigma_{R}^{k l}-\Sigma_{C}^{k l}+\Sigma_{R C}^{k l}\right| \leq \varepsilon
$$

is possible reducing the left-hand-side to the linear combination of

$$
\left|a_{i j}^{k l}\right|, \quad \sum_{i=1}^{\infty}\left|\triangle_{10} a_{i j}^{k l}\right|, \quad \sum_{j=1}^{\infty}\left|\triangle_{01} a_{i j}^{k l}\right|, \quad \sum_{i=1}^{\infty} \sum_{j=1}^{\infty}\left|\square a_{i j}^{k l}\right| .
$$

By Corollary 2.4 and Lemma $2.1(v) \in \mathbf{b},\left(v_{\square}\right) \in \mathbf{b c n}$ imply $(i),\left(i v_{\triangle}\right) \in \mathbf{b c n}$. Estimates by using $\sum_{i j}\left|\square a_{i j}\right|$ are given in [3]. For a fixed $\nu$

$$
\frac{1}{p q} \sum_{m=i-p+1}^{i-2} \sum_{n=j-q+1}^{j-1} \sum_{\mu=m}^{i-1} \sum_{\nu=n}^{j-1}\left|\square a_{\mu \nu}\right|
$$


is a sum over a triangle with vertices

$$
(i-p+1, i-p+1), \quad(i-1, i-p+1), \quad(i-1, i-1) .
$$

It is dominated by the sum over the rectangle with vertices $(3.22)$ plus $(i-$ $p+1, i-1)$ :

$$
\sum_{m=i-p+1}^{i-1} \sum_{\mu=1-p+1}^{i-1}\left|\square a_{\mu \nu}\right|=(p-1) \sum_{\mu=1-p+1}^{i-1}\left|\square a_{\mu \nu}\right| .
$$

The same operation on the plane $(\nu, n)$ extends (3.23) to

$$
(p-1)(q-1) \sum_{\mu=1-p+1}^{i-1} \sum_{\nu=j-q+1}^{j-1}\left|\square a_{\mu \nu}\right| .
$$

As the result, the corresponding term of (3.17) is dominated by

$$
\begin{gathered}
\frac{(p-1)(q-1)}{p q} \sup _{i j}\left|x_{i j}\right| \sum_{i=p}^{\infty} \sum_{j=q}^{\infty} \sum_{\mu=i-p+1}^{i-1} \sum_{\nu=j-q+1}^{j-1}\left|\square a_{\mu \nu}\right| \\
\leq \frac{(p-1)^{2}(q-1)^{2}}{p q} \sup _{i j}\left|x_{i j}\right| \sum_{i=1}^{\infty} \sum_{j=1}^{\infty}\left|\square a_{i j}\right|
\end{gathered}
$$

as it is in $[3$, p. 259, (30)].

REMARK 3.3. The proof of [3, Theorem 1, p. 256] has few obvious misprints. E.g., at p. $257\left\lfloor 1(24)\right.$ the bracket must be $\left(a_{m j}-a_{i j}\right)$; p. $258\lceil 4(25)$ instead of $\frac{1}{p}$ must be $\frac{1}{q}$; p. $261\left\lceil 1\right.$ instead of $\in$ must be $\notin ;\left\lceil 9(33)\right.$ after $a_{i j}^{k_{r}, l_{r}}$ must stay $x_{i j}$, the sum $\left\lceil 10\right.$ must be $2 \sum_{i=1}^{\infty} \sum_{j=1}^{2 n_{r-1}}$, and instead of $=$ must be $\geq$. Similarly, at p. 263 , (40)(d) must start with $\lim _{k, l} \sum_{j}\left|\triangle \alpha_{j}^{k l}\right|$; the words rows at $\lceil 22$ and columns at $\lceil 24$ must be canceled. Instead of [12]「15, [11]「16 at p. 255 must be [13], [12].

Lemma 3.4. Assume that $\left(x_{i j}\right)$ a-converges to 0 . Then, sufficient conditions for $\left|\sum_{R}^{k l}\right| \leq \varepsilon$ for a given $\varepsilon>0$ as $p, q, k, l \rightarrow \infty$ are

$$
(v) \in \mathbf{b} ; \quad\left(v_{\triangle}\right)_{1} \in \mathbf{b c n} .
$$

Similarly, a sufficient condition for $\left|\Sigma_{C}^{k l}\right| \leq \varepsilon$ as $p, q, k, l \rightarrow \infty$ in case that $\left(x_{i j}\right)$ a-converges to 0 is

$$
(v) \in \mathbf{b} ; \quad\left(v_{\triangle}\right)_{2} \in \mathbf{b c n} .
$$

Proof. We start with

$$
\Sigma_{R}^{k l}=\sum_{R C}^{k l}-\left(\sum_{R C}^{k l}-\sum_{R}^{k l}\right) .
$$


The part of (3.28) in the brackets is

(3.29) $\Sigma_{R C}^{k l}-\Sigma_{R}^{k l}=\frac{1}{p q} \sum_{n=1}^{\infty} \sum_{m=1}^{\infty} a_{m n}^{k l}\left(\sum_{j=n}^{n+q-1} \sum_{i=m}^{m+p-1} x_{i j}-q \sum_{i=m}^{m+p-1} x_{i n}\right)$.

The $\sum_{R C}^{k l}$ in (3.29) we transform as follows:

$$
\begin{aligned}
\Sigma_{R C}^{k l}= & \frac{1}{p q} \sum_{m=1}^{\infty} \sum_{n=1}^{\infty} a_{m n}^{k l}\left(\sum_{i=m}^{m+p-1} x_{i n}+\cdots+\sum_{i=m}^{m+p-1} x_{i, n+q-1}\right) \\
= & \frac{1}{p q} \sum_{m=1}^{\infty} a_{m 1}^{k l}\left(\sum_{i=m}^{m+p-1} x_{i 1}+\cdots+\sum_{i=m}^{m+p-1} x_{i, q-1}+\sum_{i=m}^{m+p-1} x_{i q}\right) \\
& +\cdots \\
& +\frac{1}{p q} \sum_{m=1}^{\infty} a_{m q}^{k l}\left(\sum_{i=m}^{m+p-1} x_{i q}+\cdots+\sum_{i=m}^{m+p-1} x_{i, 2 q-1}\right) \\
& +\cdots \\
= & \frac{1}{q} \sum_{m=1}^{\infty} a_{m 1}^{k l} \frac{1}{p} \sum_{i=m}^{m+p-1} x_{i 1}+\frac{1}{q} \sum_{m=1}^{\infty}\left(a_{m 1}^{k l}+a_{m 2}^{k l}\right) \frac{1}{p} \sum_{i=m}^{m+p-1} x_{i 2}+\cdots \\
& +\frac{1}{q} \sum_{m=1}^{\infty}\left(a_{m 1}^{k l}+\cdots+a_{m, q-1}^{k l}\right) \frac{1}{p} \sum_{i=m}^{m+p-1} x_{i, q-1} \\
& +\frac{1}{q} \sum_{m=1}^{\infty} \sum_{n=1}^{\infty}\left(a_{m n}^{k l}+\cdots+a_{m, n+q-1}^{k l}\right) \frac{1}{p} \sum_{i=m}^{m+p-1} x_{i, n+q-1} .
\end{aligned}
$$

Denoting the sum of the first $q-1$ lines above by $\Sigma_{R C}^{k l}(I)$, and the last line by

$$
\Sigma_{R C}^{k l}(I I)=\frac{1}{p q} \sum_{m=1}^{\infty} \sum_{n=1}^{\infty}\left(a_{m n}^{k l}+\cdots+a_{m, n+q-1}^{k l}\right) \sum_{i=m}^{m+p-1} x_{i, n+q-1}
$$

we obtain

$$
\Sigma_{R C}^{k l}=\Sigma_{R C}^{k l}(I)+\Sigma_{R C}^{k l}(I I) .
$$

Splitting the sum with respect to $j$ of $\Sigma_{R}^{k l}$ in (3.29) for the part with $j \leq q-1$ and the part with $j \geq q$ it follows that

$$
\begin{aligned}
& \sum_{R}^{k l}(I)=\frac{1}{p q} \sum_{m=1}^{\infty} \sum_{j=1}^{q-1} a_{m j}^{k l} q \sum_{i=m}^{m+p-1} x_{i j}, \\
& \Sigma_{R}^{k l}(I I)=\frac{1}{p q} \sum_{m=1}^{\infty} \sum_{j=q}^{\infty} a_{m j}^{k l} q \sum_{i=m}^{m+p-1} x_{i j} .
\end{aligned}
$$


Therefore

$$
\begin{aligned}
\Sigma_{R}^{k l}= & \sum_{R}^{k l}(I)+\sum_{R}^{k l}(I I) \\
= & \sum_{m=1}^{\infty} a_{m 1}^{k l} \frac{1}{p} \sum_{i=m}^{m+p-1} x_{i j}+\cdots \\
& +\sum_{m=1}^{\infty} a_{m, q-1}^{k l} \frac{1}{p} \sum_{i=m}^{m+p-1} x_{i, q-1}+\frac{1}{p q} \sum_{m=1}^{\infty} q \sum_{j=q}^{\infty} a_{m j}^{k l} \sum_{i=m}^{m+p-1} x_{i j}
\end{aligned}
$$

with

$$
\begin{aligned}
\sum_{R}^{k l}(I I) & =\frac{1}{p q} \sum_{m=1}^{\infty} \sum_{j=q}^{\infty} a_{m j}^{k l} q \sum_{i=m}^{m+p-1} x_{i j} \\
& =\frac{1}{p q} \sum_{m=1}^{\infty} \sum_{n=1}^{\infty} q a_{m, n+q-1}^{k l} \sum_{i=m}^{m+p-1} x_{i, n+q-1}
\end{aligned}
$$

We estimate (3.28) by

$$
\begin{aligned}
\left|\Sigma_{R}^{k l}\right| & \leq\left|\sum_{R C}^{k l}\right|+\left|\Sigma_{R C}^{k l}-\Sigma_{R}^{k l}\right| \\
& \leq\left|\sum_{R C}^{k l}\right|+\left|\Sigma_{R C}^{k l}(I)\right|+\left|\Sigma_{R}^{k l}(I)\right|+\left|\sum_{R C}^{k l}(I I)-\Sigma_{R}^{k l}(I I)\right| .
\end{aligned}
$$

Now we look at members of (3.36). First, we take $N_{\varepsilon}$ defined by (1.6) and $C$ defined by (2.2). For $p, q \geq N_{\varepsilon}$ we have

$$
\left|\Sigma_{R C}^{k l}\right|=\left|\sum_{n=1}^{\infty} \sum_{m=1}^{\infty} a_{m n}^{k l} \frac{1}{p q} \sum_{j=n}^{n+q-1} \sum_{i=m}^{m+p-1} x_{i j}\right| \leq C \varepsilon .
$$

For a particular $n$ and $\varepsilon>0$, by (2.20) and a sufficiently large $k, l$,

$$
\sum_{m=1}^{\infty}\left|a_{m n}^{k l}\right| \leq \frac{\varepsilon}{q-1}
$$

for every $n$ (Corollary 2.5 and Remark 2.8).

By (3.30), $\left|\sum_{R C}^{k l}(I)\right|$ is estimated from above by

$$
\begin{gathered}
(q-1) \sum_{m=1}^{\infty}\left|a_{m 1}^{k l}\right| \frac{1}{q}\left|\frac{1}{p} \sum_{i=m}^{m+p-1} x_{i 1}\right|+\ldots \\
\cdots+\sum_{m=1}^{\infty}\left|a_{m, q-1}^{k l}\right| \frac{1}{q}\left|\frac{1}{p} \sum_{i=m}^{m+p-1} x_{i, q-1}\right|
\end{gathered}
$$


By (3.38) and choosing $k, l$ large enough we obtain

$$
\sum_{i=1}^{\infty}\left|a_{i j}^{k l}\right|<\frac{\varepsilon}{q-1}, \quad j=1, \ldots, q-1 .
$$

With

$$
\sup _{i j}\left|x_{i j}\right|=B<+\infty
$$

we have

$$
\frac{1}{q} \sum_{m=1}^{\infty}\left|a_{m j}^{k l}\right|\left|\frac{1}{p} \sum_{i=m}^{m+p-1} x_{i j}\right| \leq \frac{1}{q} B \frac{\varepsilon}{q-1}, \quad j=1, \ldots, q-1
$$

for every $p \in \mathbb{N}$. Estimating (3.39) we obtain

$$
\left|\Sigma_{R C}^{k l}(I)\right| \leq \frac{(q-1) q}{2} \cdot \frac{1}{q} B \frac{\varepsilon}{q-1}=\frac{B \varepsilon}{2} .
$$

The estimate for $\sum_{R}^{k l}(I)$ in (3.33) differs from (3.39) having no coefficients $\frac{q-1}{q}, \ldots, \frac{1}{q}$. Therefore

$$
\left|\sum_{R}^{k l}(I)\right| \leq B \varepsilon
$$

To complete the estimate of (3.36) we estimate the difference of (3.31) and (3.35):

$$
\begin{aligned}
\sum_{R C}^{k l}(I I)-\sum_{R}^{k l}(I I) \\
=\frac{1}{q} \sum_{n=1}^{\infty} \sum_{m=1}^{\infty}\left(\left(a_{m n}^{k l}-a_{m, n+q-1}^{k l}\right)\right. \\
\left.\quad+\cdots+\left(a_{m, n+q-1}^{k l}-a_{m, n+q-1}^{k l}\right)\right) \frac{1}{p} \sum_{i=m}^{m+p-1} x_{i, n+q-1} .
\end{aligned}
$$

For $q \geq 2$ the above differences are sums

$$
\begin{aligned}
& \left(a_{m n}^{k l}-a_{m, n+1}^{k l}\right)+\cdots+\left(a_{m, n+q-2}^{k l}-a_{m, n+q-1}^{k l}\right) \\
& \left(a_{m, n+1}^{k l}-a_{m, n+2}^{k l}\right)+\cdots+\left(a_{m, n+q-2}^{k l}-a_{m, n+q-1}^{k l}\right)
\end{aligned}
$$

with $q-1, q-2, \ldots, 1$ terms. For every $m$ in (3.46) there are $\frac{(q-1) q}{2}$ terms with $\Delta_{01} a_{m j}^{k l}$ (counting separately members that in the sum of lines (3.46) result to be equal).

The $\left(v_{\triangle}\right)_{1} \in \mathbf{b c n}$ in (3.26) means that

$$
\lim _{k, l} \sum_{n=1}^{\infty} \sum_{m=1}^{\infty}\left|\left(a_{m n}^{k l}-a_{m, n+1}^{k l}\right)\right|=0
$$


The same equality is valid if we have $\triangle_{01} a_{m, n+\varrho}^{k l}(\varrho=0,1, \ldots, q-2)$ instead of $\triangle_{01} a_{m n}^{k l}$. The (3.46) is reduced to the sum of

$$
\frac{1}{q} \sum_{n=1}^{\infty}\left(\sum_{m=1}^{\infty} \triangle_{01} a_{m, n+\varrho}^{k l} \frac{1}{p} \sum_{i=m}^{m+p-1} x_{i, n+q-1}\right)
$$

with $\varrho=0, \ldots, q-2$. The absolute value of $(3.48)$ is less or equal to

$$
\frac{1}{q} \sum_{n=1}^{\infty}\left|\sum_{m=1}^{\infty} \triangle_{01} a_{m, n+\varrho}^{k l}\right| B,
$$

where by $B=\sup _{i j}\left|x_{i j}\right|$. By the $\frac{\varepsilon}{q-1}$-estimate for (3.47) the (3.49) is $\leq \frac{B \varepsilon}{q(q-1)}$. Therefore (3.45) is estimated by

$$
\left|\sum_{R C}^{k l}(I I)-\sum_{R}^{k l}(I I)\right| \leq \frac{B \varepsilon}{2} .
$$

By (3.50), (3.43) and (3.44)

$$
\left|\sum_{R C}^{k l}(I I)-\sum_{R}^{k l}(I I)\right|+\left|\sum_{R C}^{k l}(I)\right|+\left|\Sigma_{R}^{k l}(I)\right| \leq 2 B \varepsilon .
$$

Therefore, with (3.37) we have the estimate for (3.36):

$$
\left|\sum_{R}^{k l}\right| \leq(C+2 B) \varepsilon
$$

This means that Lemma 3.4 holds for $\Sigma_{R}^{k l}$. Similarly for $\Sigma_{C}^{k l}$.

ThEOREM 3.5. The matrix $A=\left[a_{i j}^{k l}\right]$ transforms the space racrn into the space bcn if and only if

$$
(v) \in \mathbf{b} ; \quad\left(v_{\triangle}\right) \in \mathbf{b c n} .
$$

Proof. Sufficiency. With (3.10) and (3.11), $y^{k l}$ is estimated by estimates for $Y^{k l}, \Sigma_{R C}^{k l}, \Sigma_{R}^{k l}$ and $\Sigma_{C}^{k l}$. Let $p, q$ be such that $\left|\sum_{R C}^{k l}\right|<\varepsilon C$, which is possible by the existence of the $P$ a-limit 0 of $\sigma_{p q}^{m n}$ and $C$ from (2.2). Lemma 3.2 gives $Y^{k l}$ as small as we please with conditions $(v) \in \mathbf{b}$ and $\left(v_{\square}\right) \in$ bcn. For $\Sigma_{R}^{k l}$ and $\Sigma_{C}^{k l}$, conditions on $\left(v_{\triangle}\right)_{1}$, resp. $\left(v_{\triangle}\right)_{2}$, are given by Lemma 3.4. Conditions (3.26) and (3.27) assure that, by $k, l \rightarrow \infty$ and every $x \in$ racrn, both $\Sigma_{R}^{k l}$ and $\Sigma_{C}^{k l}$ converge to 0 . The same conditions imply $\left(v_{\square}\right) \in \mathbf{b c n}$ (Lemma 2.7). Therefore $y^{k l}$ from (3.10) also $P$ converges to 0 .

Necessity. We assume $(v) \in \mathbf{b}$ and $(i) \in \mathbf{b c n}$ which are N.S. for the transformation of rcrn to bcn. The necessity of $\left(v_{\triangle}\right)_{1} \in \mathbf{b c n}$ for the space of all double sequences with columns uniformly $a$-convergent to 0 and rows nonuniformly $a$-convergent to 0 is proved in [12, pp. 287-288]. The proof there is given for uniformly $a$-convergent rows. Moreover, the bounded regularity of 
$\left[a_{m n}^{k l}\right]$ is assumed. It includes $(i v) \in \mathbf{b c n}$ which is not needed for the necessity proof but follows from $\left(v_{\triangle}\right) \in \mathbf{b c n}$ by Corollary 2.5 .

REMARK 3.6. The sufficiency proof in [12] uses $\left(v_{\triangle}\right)_{1}$ and $\left(v_{\triangle}\right)_{2}$ together. In our setting they are related to $\Sigma_{R}^{k l}$, resp. to $\Sigma_{C}^{k l}$. In the case of the uniformly a-convergent columns $\Sigma_{C}^{k l}$ is small for large $q$ and $Y^{k l}$ is estimated via Lemma 2.7. Notice that the $p$ and $q$ are determined by (3.37) and hereafter remain fixed.

COROllary 3.7. Let subspaces of racrn be sets of all $\left(x_{i j}\right)$ such that their a-convergence to 0 is
i. uniform by rows and columns
ii. uniform by columns
iii. uniform by rows
iv. without uniformity restrictions.

Conditions on $\left[a_{i j}^{k l}\right]$, N.S. for the transformation of the classes above into bcn, corresponding to the respective cases are

$$
\begin{aligned}
i^{\prime} .(v) & \in \mathbf{b}, \quad\left(v_{\square}\right) \in \mathbf{b c n} \\
i i^{\prime} .(v) & \in \mathbf{b}, \quad\left(v_{\triangle}\right)_{1} \in \mathbf{b c n} \\
i i i^{\prime} .(v) & \in \mathbf{b}, \quad\left(v_{\triangle}\right)_{2} \in \mathbf{b c n} \\
i v^{\prime} .(v) & \in \mathbf{b}, \quad\left(v_{\triangle}\right) \in \mathbf{b c n} .
\end{aligned}
$$

TheOREM 3.8. The matrix $A=\left[a_{i j}^{k l}\right]$ transforms the space rac into the space bc such that $\lim _{k l} y^{k l}$ of $y=A x$ is equal to $\operatorname{Lim}_{i j} x^{i j}$ if and only if

$$
(\text { iii }) \in \mathbf{b c} \quad \lim _{k l}=1 ; \quad(v) \in \mathbf{b} ; \quad\left(v_{\triangle}\right) \in \mathbf{b c n} .
$$

Proof. Sufficiency. We estimate

$$
y^{k l}-L=Y^{k l}-\left(\Sigma_{R C}^{k l}-L\right)+\left(\Sigma_{R}^{k l}-L\right)+\left(\Sigma_{C}^{k l}-L\right) .
$$

By the Lemma 3.2, $(v) \in \mathbf{b}$ and $\left(v_{\square}\right) \in \mathbf{b c n}$ are sufficient for $\left|Y^{k l}\right| \leq \varepsilon$ if $k, l$ are sufficiently large. 
The last three terms of (3.57) are

$$
\begin{aligned}
\sum_{R C}^{k l}-L= & \sum_{m=1}^{\infty} \sum_{n=1}^{\infty} a_{m n}^{k l}\left[\frac{1}{p q} \sum_{i=m}^{m+p-1} \sum_{j=n}^{n+q-1}\left(x_{i j}-L\right)\right] \\
& +\left(\sum_{m=1}^{\infty} \sum_{n=1}^{\infty} a_{m n}^{k l}-1\right) L \\
\sum_{R}^{k l}-L= & \sum_{m=1}^{\infty} \sum_{j=1}^{\infty} a_{m j}^{k l}\left[\frac{1}{p} \sum_{i=m}^{m+p-1}\left(x_{i j}-L_{i}^{\prime \prime}\right)+\left(\frac{1}{p} \sum_{i=m}^{m+p-1} L_{i}^{\prime \prime}-L\right)\right] \\
& +\left(\sum_{m=1}^{\infty} \sum_{n=1}^{\infty} a_{m n}^{k l}-1\right) L, \\
& +\sum_{i=1}^{\infty} \sum_{n=1}^{\infty} a_{i n}^{k l}\left[\frac{1}{q} \sum_{j=n}^{n+q-1}\left(x_{i j}-L_{j}^{\prime}\right)+\left(\frac{1}{q} \sum_{j=n}^{n+q-1} L_{j}^{\prime}-L\right)\right] \\
& \left(\sum_{m=1}^{\infty} \sum_{n=1}^{\infty} a_{m n}^{k l}-1\right) L .
\end{aligned}
$$

By $($ iii $) \in \mathbf{b c}, \lim _{k l}=1$, increasing $k, l$ we have $\left|\sum_{m=1}^{\infty} \sum_{n=1}^{\infty} a_{m n}^{k l}-1\right||L| \leq \frac{\varepsilon}{8}$. With $C$ from $(v) \in \mathbf{b}$ and the $P a$-convergence of $x$, the choice of $p, q$ gives $\left|\sigma_{p q}^{m n}-L\right| \leq \frac{\varepsilon}{8 C}$ and $\left|\sum_{R C}^{k l}-L\right| \leq \frac{\varepsilon}{4}$. By Lemma 3.1, $\left|\frac{1}{p} \sum_{i=m}^{m+p-1} L_{i}^{\prime \prime}-L\right|$ and $\left|\frac{1}{q} \sum_{j=n}^{n+q-1} L_{j}^{\prime}-L\right|$ are also $\leq \frac{\varepsilon}{8 C}$. The terms $\left(x_{i j}-L_{i}^{\prime \prime}\right) \operatorname{resp} .\left(x_{i j}-L_{j}^{\prime}\right)$ in $\sum_{R}^{k l}-L$ and $\sum_{C}^{k l}-L$ a-converge to 0 . Lemma 3.4 applies on $x_{i j}-L_{i}^{\prime \prime}$ because $\left(x_{i j}\right)$ and $\left(L_{i}^{\prime \prime}\right)$ as double sequences $a$-converge to $L: x \in$ rac and $\frac{1}{p} \sum_{i=m}^{m+p-1} \frac{1}{q} \sum_{j=n}^{n+q-1} L_{i}^{\prime \prime}=\frac{1}{p} \sum_{i=m}^{m+p-1} L_{i}^{\prime \prime}$ converges to $L$ if $p, q \rightarrow \infty$. By (3.26) and (3.27) i.e. by $(v) \in \mathbf{b}$ and $\left(v_{\triangle}\right)_{1} \in \mathbf{b c n}$, resp. $\left(v_{\triangle}\right)_{2} \in \mathbf{b c n}$, if $k, l \rightarrow \infty$, the absolute value of these members becomes $\leq \frac{\varepsilon}{8}$. Therefore, the linear combination of (3.58) is by absolute value $\leq \varepsilon$ and $\left|y^{k l}-L\right| \leq 2 \varepsilon$.

Necessity. The N.S. conditions for the a-convergence of sequences which have uniformly $a$-convergent rows and columns (the class rac un denoted in [3] by arc) are necessary conditions for transformations of sequences from rac. This means that $(v) \in \mathbf{b},(i i i) \in \mathbf{b c}$ with $\lim _{k l}=1$ and $\left(v_{\square}\right) \in \mathbf{b c n}$ are necessary. Other conditions, given in [3, Theorem 2, p. 361] follow by implications (2.23). Also, necessary are $\left(v_{\triangle}\right) \in \mathbf{b c n}$ because of racrn $\subset$ rac and the Theorem 3.5 above.

Similarly to Corollary 3.7, subclasses of rac are characterized by uniformities of row, resp. column, $a$-convergence. 
COROLlary 3.9. Let subspaces of rac be sets of all $\left(x_{i j}\right)$ such that their a-convergence is

$$
\begin{aligned}
& \text { i.uniform by rows and columns } \\
& \text { ii.uniform by columns } \\
& \text { iii.uniform by rows } \\
& \text { iv.without uniformity restrictions. }
\end{aligned}
$$

Conditions on A, N.S. that $y=A x$ belongs to bc with $\lim _{k l} y^{k l}=\operatorname{Lim}_{i j} x_{i j}$ are

$$
(\text { iii }) \in \mathbf{b c}, \quad \lim _{k l}=1, \quad(v) \in \mathbf{b}
$$

and, moreover, for cases $i-i v$,

$$
\begin{aligned}
& i^{\prime} \cdot\left(v_{\square}\right), \quad\left(v i_{\triangle}\right) \in \mathbf{b c n} \\
& i i^{\prime} \cdot\left(v_{\triangle}\right)_{1}, \quad\left(v i_{\triangle}\right)_{2} \in \mathbf{b c n} \\
& i i i^{\prime} \cdot\left(v_{\triangle}\right)_{2}, \quad\left(v i_{\triangle}\right)_{1} \in \mathbf{b c n} \\
& i v^{\prime} \cdot\left(v_{\triangle}\right) \in \mathbf{b c n} .
\end{aligned}
$$

Proof. The proof for $i$ is given in [3]. We look at the proof for $i i$. Sufficiency. For $\sum_{R}^{k l}-L$ in (3.58) we apply (3.60) and $\left(v_{\triangle}\right)_{1} \in$ bcn as in the proof of Theorem 3.8. For $\sum_{C}^{k l}-L$ we have

$$
\begin{aligned}
\sum_{C}^{k l}-L= & \sum_{i=1}^{\infty} \sum_{n=1}^{\infty} a_{i n}^{k l}\left[\left(\frac{1}{q} \sum_{j=n}^{n+q-1} x_{i j}-L_{i}^{\prime \prime}\right)+\left(L_{i}^{\prime \prime}-L\right)\right] \\
& +\left(\sum_{m=1}^{\infty} \sum_{n=1}^{\infty} a_{m n}^{k l}-1\right) L .
\end{aligned}
$$

Because of the uniformity for $\varepsilon>0$ by increasing $q$ we obtain $\mid \frac{1}{q} \sum_{j=n}^{n+q-1} x_{i j}-$ $L_{i}^{\prime \prime} \mid \leq \varepsilon$ for every $n, i=1,2, \ldots$. For the transformation of $\left(L_{i}^{\prime \prime}-L\right)$ we apply $\left(v i_{\triangle}\right)_{2} \in \mathbf{b c n}$.

Necessity. For $\Sigma_{C}^{k l}$ and $Y^{k l}$ transformation conditions follow from [3, Theorem 2, p. 261] where N.S. conditions are given for the case $i$. Conditions $(i),(i i) \in \mathbf{b c n}$ in [3] are superfluous by Lemmas 2.2 and 2.3. For $\Sigma_{R}^{k l}$, as for Theorems above, necessity follows from the proof for $y^{k l}$ in [12]. MóriczRhoades conditions for transformations of the class ac in [12] are conditions of our Theorem 3.8. For the transformations of acn the $($ iii $) \in \mathbf{b}, \lim _{k l}=1$ in [12] is superfluous (cf. also [4, 35. $R C N \rightarrow B C N$, p. 49]). Conditions that follow from [12] for transformations of acn are the same as our conditions for transformations of racrn and therefore also for our racn (Theorem 3.5). 
Proofs of the cases (ii) and (iii) in Corollaries 3.7 and 3.9 remain valid without the $a$-convergence of rows, resp. columns (see Remark 3.6). We denote subspaces of acn and of ac with $H$ a-convergence by columns, resp. by rows, by $\mathbf{r}_{1}$ acn and $\mathbf{r}_{1}$ ac, resp. by $\mathbf{r}_{2}$ acn and $\mathbf{r}_{2}$ ac. Therefore, $\mathbf{r}_{1}$ ac is the class of sequences with the $P a$-limit $L$ and with columns that $a$-converge to $L_{i}^{\prime \prime}$. By Lemma 1.1 the $L_{i}^{\prime \prime} a$-converges to $L$ but row $a$-limits don't necessarily exist. The class $\mathbf{r}_{\mathbf{1}}$ acr is a subclass of $\mathbf{r}_{\mathbf{1}}$ ac with $L_{i}^{\prime \prime}=L$ for every $i$ and $\mathbf{r}_{\mathbf{1}}$ acn is a subclass of $\mathbf{r}_{1}$ ac with $L=0$. For $x \in \mathbf{r}_{1}$ acn and $i \in \mathbb{N}$ the $L_{i}^{\prime \prime}$ are not necessarily 0 . The class $\mathbf{r}_{1}$ acrn is a subclass of $\mathbf{r}_{1}$ ac with $L_{i}^{\prime \prime}=L=0$ for every $i$. If the $a$-convergence of columns to $L_{i}^{\prime \prime}$ is uniform, to the designation of the class we add un. If instead of columns we have the $a$-convergence by rows, instead of $\mathbf{r}_{1}$ we have $\mathbf{r}_{2}$.

Because the classes $\mathbf{r}_{\mathbf{1}}$ acrn and $\mathbf{r}_{\mathbf{1}}$ ac as well as $\mathbf{r}_{\mathbf{2}}$ acrn and $\mathbf{r}_{\mathbf{2}}$ ac are in the between of racrn and ac, N.S. conditions for transformations of these classes to bcn and bc are conditions of Theorems 3.5 and 3.8. In case that the $H a$-convergence by columns or by rows is uniform, the corresponding conditions differ from conditions [12].

THEOREM 3.10. The matrix $\left[a_{i j}^{k l}\right]$ transforms the space $\mathbf{r}_{1}$ acrn un into the space bcn if and only if

$(v) \in \mathbf{b}, \quad\left(v_{\triangle}\right)_{1} \in \mathbf{b c n}$.

Conditions N.S. for the transformation of $\mathbf{r}_{\mathbf{2}}$ acrn un into bcn are

$(v) \in \mathbf{b}, \quad\left(v_{\triangle}\right)_{2} \in \mathbf{b c n}$.

The matrix $A=\left[a_{i j}^{k l}\right]$ transforms $\mathbf{r}_{1}$ ac un into bc such that $\lim _{k l} y^{k l}$ of $y=A x$ is equal to $\operatorname{Lim}_{i j} x_{i j}$ if and only if

(3.65) $\quad(i i i) \in \mathbf{b c}, \lim _{k l}=1, \quad(v) \in \mathbf{b}, \quad\left(v_{\triangle}\right)_{1} \in \mathbf{b c n}, \quad\left(v i_{\triangle}\right)_{2} \in \mathbf{b c n}$.

The N.S. conditions for the analogous transformation of $\mathbf{r}_{\mathbf{2}}$ ac un are

$$
(\text { iii }) \in \mathbf{b c}, \lim _{k l}=1, \quad(v) \in \mathbf{b}, \quad\left(v_{\triangle}\right)_{2} \in \mathbf{b c n}, \quad\left(v i_{\triangle}\right)_{1} \in \mathbf{b c n} .
$$

The N.S. conditions on $A$ for the transformation of $\mathbf{r}_{1}$ acr un, resp. $\mathbf{r}_{2} \mathbf{a c r}$ un, into bc with $\lim _{k l} y_{k l}=\operatorname{Lim}_{i j} x_{i j}$ are (3.63) resp. (3.64), with added $($ iii $) \in \mathbf{b c}, \lim _{k l}=1$.

The N.S. conditions on A for the transformation of $\mathbf{r}_{\mathbf{1}} \mathbf{a c n} \mathbf{u n , ~ r e s p . ~}$ $\mathbf{r}_{2}$ acn un, into bcn are (3.65), resp. (3.66) without $(i i i) \in \mathbf{b c}, \lim _{k l}=1$.

Proof. With inclusions commented in the introduction of classes with $\mathbf{r}_{\mathbf{1}}$ and $\mathbf{r}_{\mathbf{2}}$ the various cases above are deduced by the corresponding cases in Corollaries 3.7 and 3.9. See also Corollary in [3, p. 261].

REMARK 3.11. In accordance with the notation for classes in Theorem 3.10 we can denote classes in Corollaries 3.7 (ii), (iii) and 3.9 (ii), (iii) by racrn $u_{1}, \operatorname{racrn} \mathbf{u n}_{2}$ and $\operatorname{rac} \mathbf{u n}_{1}, \operatorname{rac} \mathbf{u n}_{2}$. The necessity counterexample in [12] is from racrn $\mathbf{u n}_{\mathbf{2}}$. 
ACKNOWLEDGEMENT.

The author wish to thank the referee for his careful reading of the manuscript, correcting many errors and pointing on incomplete proofs.

\section{REFERENCES}

[1] T. J. l'A. Bromwich, An introduction to the theory of infinite series, ed. 2, Cambridge 1926 (repr. Macmillan, London, 1955).

[2] D. Butković, On regular almost convergence, Math. Commun. 1 (1996), 127-137.

[3] D. Butković, On almost regular convergence, Acta Math. Hungar. 82 (1999), 249-264.

[4] H. J. Hamilton, Transformations of multiple sequences, Duke Math. J. 2 (1936), 29-60.

[5] H. J. Hamilton, On transformations of double series, Bull. Amer. Math. Soc. 42 (1936), $275-283$

[6] G. H. Hardy, On the convergence of certain multiple series, Proc. Cambridge Phil. Soc. 19 (1917), 86-95.

[7] G. G. Lorentz, A contribution to the theory of divergent sequences, Acta Math. 80 (1948), 167-180.

[8] F. M. Mears, Transformations of double sequences, Amer. J. Math. 70 (1948), 804-832.

[9] F. Móricz, On the convergence in a restricted sense of multiple series, Anal. Math. 5 (1979), 135-147.

[10] F. Móricz, Some remarks on the notion of regular convergence of multiple series, Acta Math. Hungar. 41 (1983), 161-168.

[11] F. Móricz, Extensions of the spaces $c$ and $c_{0}$ from single to double sequences, Acta Math. Hungar. 57 (1991), 129-136.

[12] F. Móricz and B. E. Rhoades, Almost convergence of double sequences and strong regularity of summability matrices, Math. Proc. Cambridge Philos. Soc. 104 (1988), 283-294.

[13] A. Pringsheim, Elementare Theorie der unendlichen Doppelreihen, Sitzung-Berichte der math.-phys. Classe der Akad. der Wissenschafften zu München 27 (1897), 101-152.

[14] В. Г. Челидзе (V. G. Chelidze), Некоторые методы суммирования двойных рядов и двойных интегралов (Russian), MR522164 83g:40001: Summability methods for double series and double integrals, Izdat. Tbilis. Univ., Tbilisi, 1977.

D. Butković

University of Zagreb

Faculty of Electrical Engineering and Computing

Unska 3, 10000 Zagreb

Croatia

E-mail: dbutkovic@gmail.com

Received: 21.12.2012.

Revised: 4.11.2013. \& 15.1.2015 